Supplement of Atmos. Chem. Phys., 20, 11607-11624, 2020

https://doi.org/10.5194/acp-20-11607-2020-supplement

(C) Author(s) 2020. This work is distributed under

the Creative Commons Attribution 4.0 License.

(c) (1)

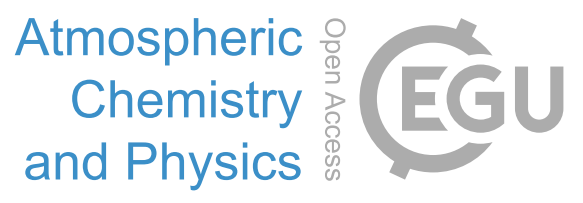

Supplement of

\title{
Differences in fine particle chemical composition on clear and cloudy days
}

Amy E. Christiansen et al.

Correspondence to: Annmarie G. Carlton (agcarlto@uci.edu)

The copyright of individual parts of the supplement might differ from the CC BY 4.0 License. 
(a)

(b)
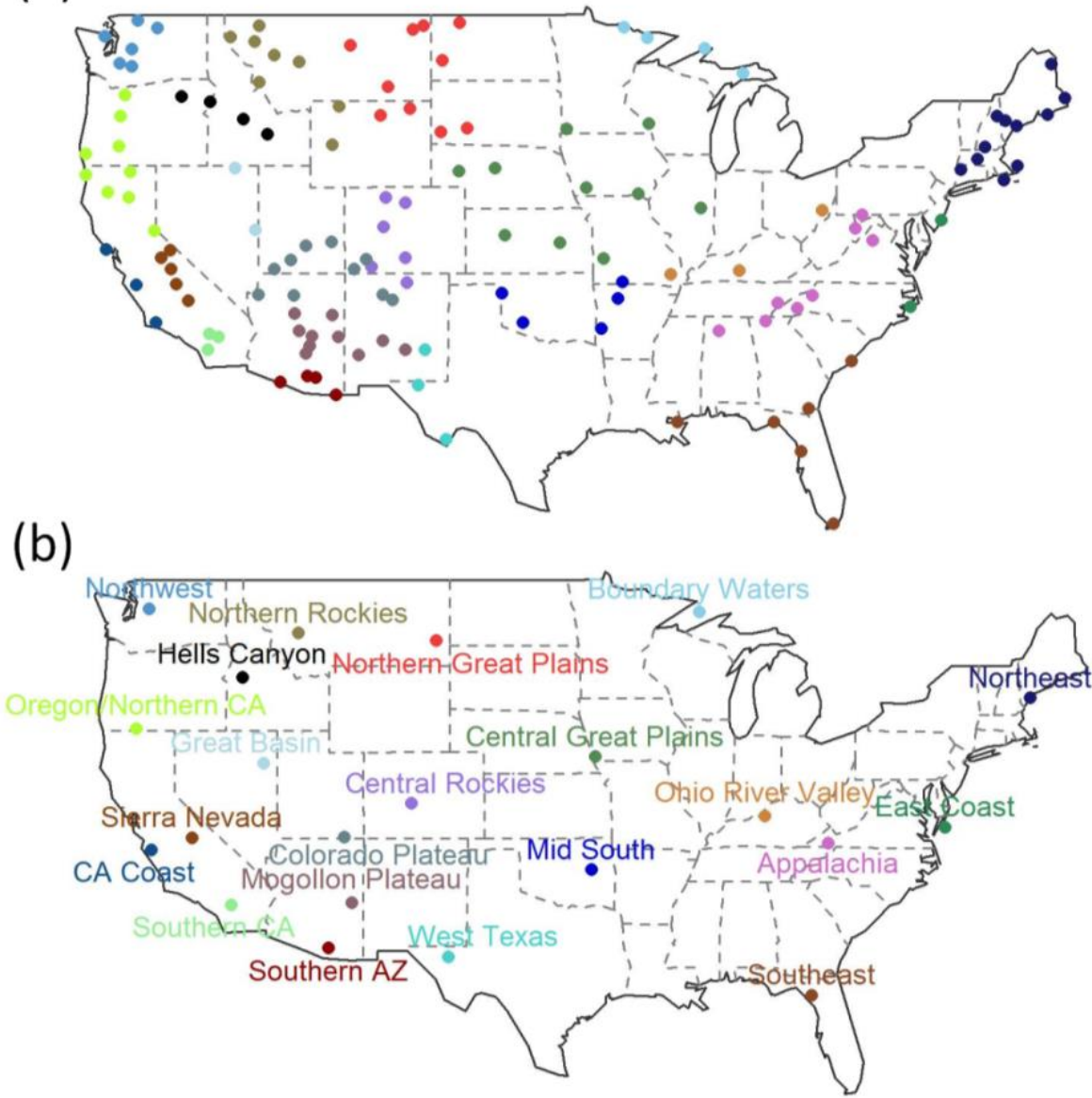

Fig. S1. Locations of all a) IMPROVE network sites and b) chemical climatology regions. Each site is color-coded by region. 


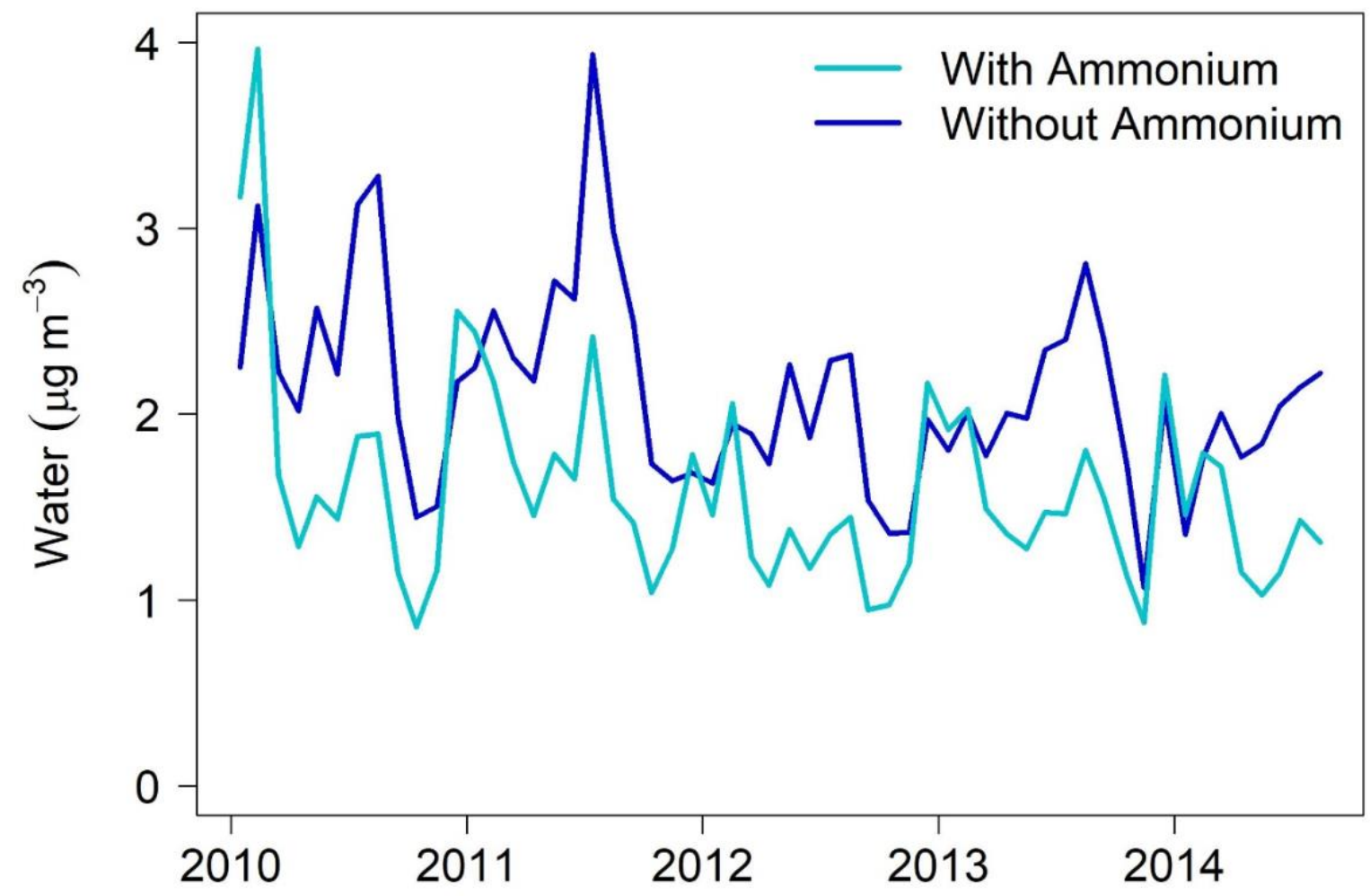

Fig. S2. ALW temporal trends with (light blue) and without (dark blue) ammonium. Ammonium measurements are not available in the IMPROVE network, but some EPA CASTNET sites are colocated with IMPROVE sites. Ammonium values are taken from 15 co-located CASTNET and IMPROVE sites, and monthly aggregates are used as inputs to ISORROPIA. Including ammonium changes absolute values but does not alter trends. 


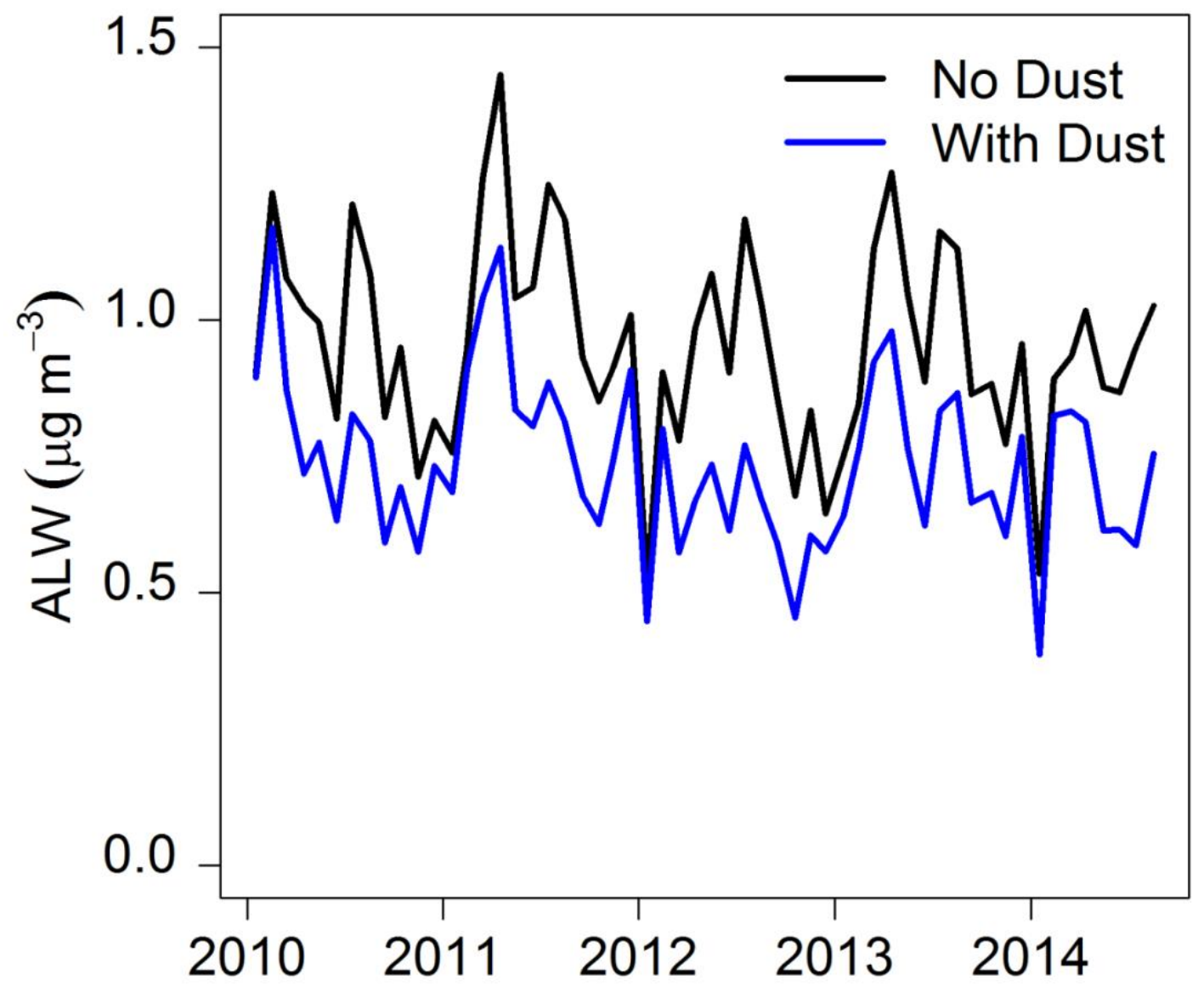

Fig. S3. Median ALW mass concentrations calculated from ISORROPIA with dust (Na+, $\mathrm{Ca}_{2}$, $\mathrm{K}_{+}, \mathrm{Mg}_{2+}, \mathrm{Cl}_{-}$) included (blue line) and without dust (black line). In the dust sensitivity, cations form insoluble species with sulfate and precipitate. If ammonium were present, it would compete with dust for sulfate and form more soluble species and increase ALW. Here, the dust sensitivity likely represents a lower bound. 


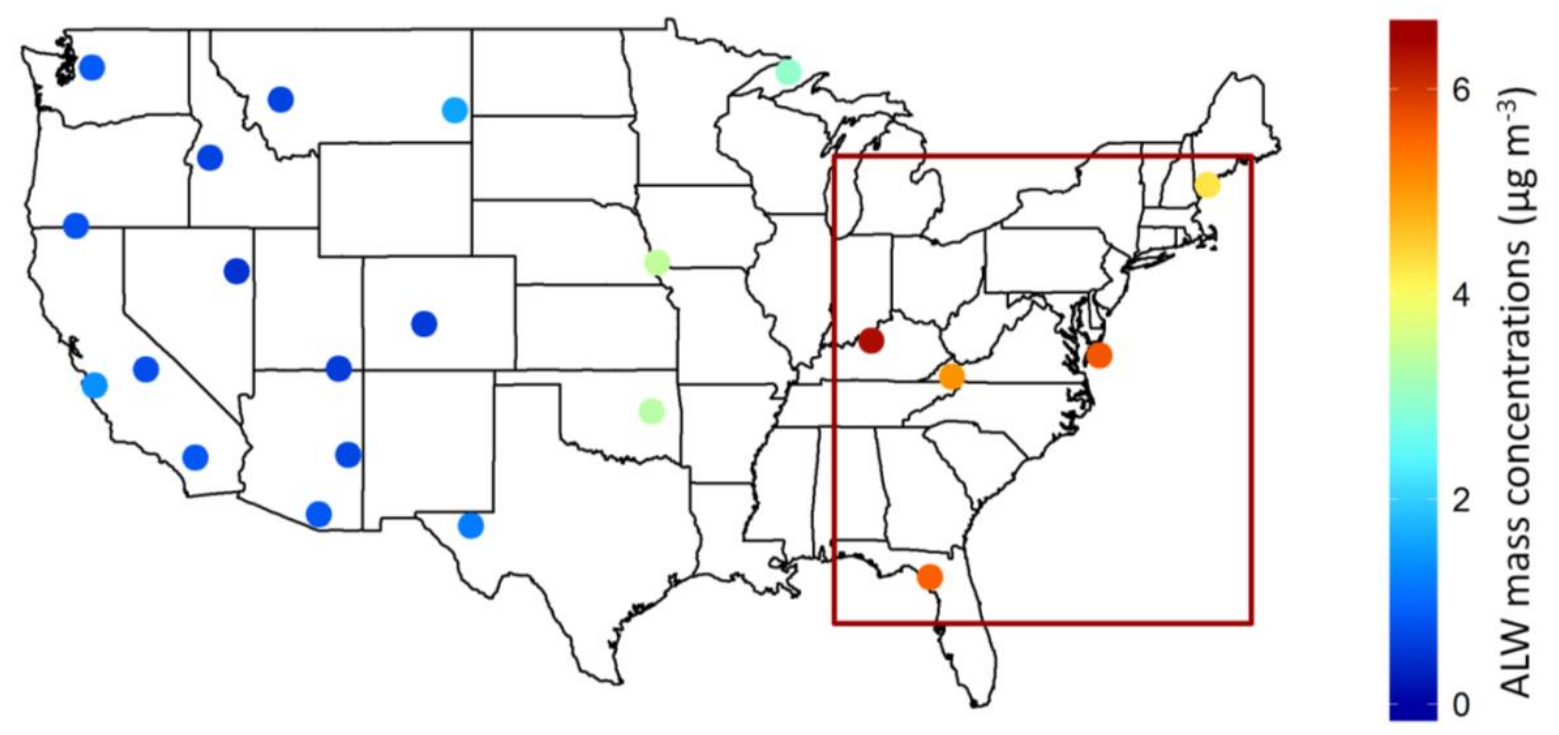

Fig. S4. Average annual ALW mass concentrations across the CONUS. The largest concentrations are located east of the Mississippi River. The boxed area indicates the chemical climatology regions investigated in the eastern US ALW scenarios. 
(a) Winter
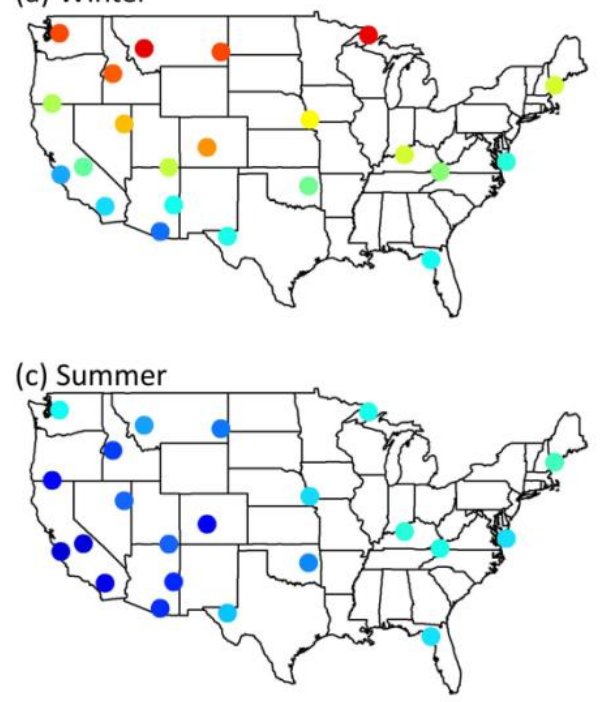

(b) Spring

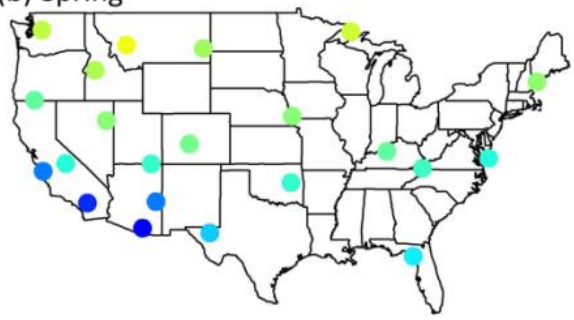

(d) Fall

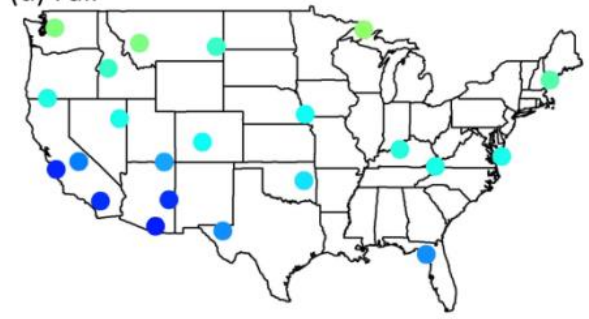

1.00

0.75 坴 0.50 案 0.25 은

Fig. S5. MODIS cloud fraction across the CONUS from 2010-2014 during a) winter, b) spring, c) summer, and d) fall. A hotter color indicates that a location is in cloud for a greater percentage of time. 


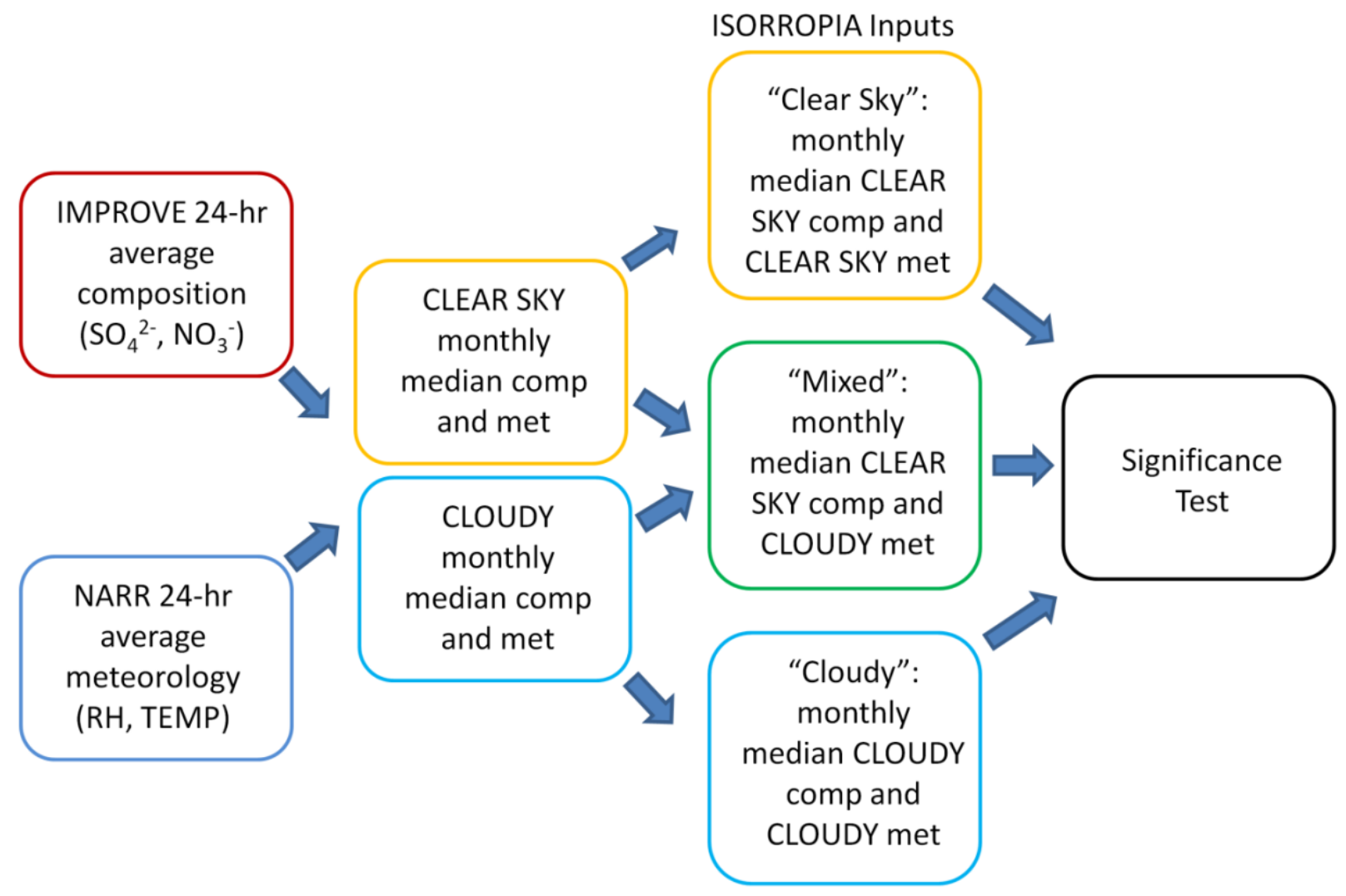

Fig. S6. Flowchart describing how the three scenarios (Cloudy, Clear Sky, and Mixed) for the eastern US are performed and compared. 


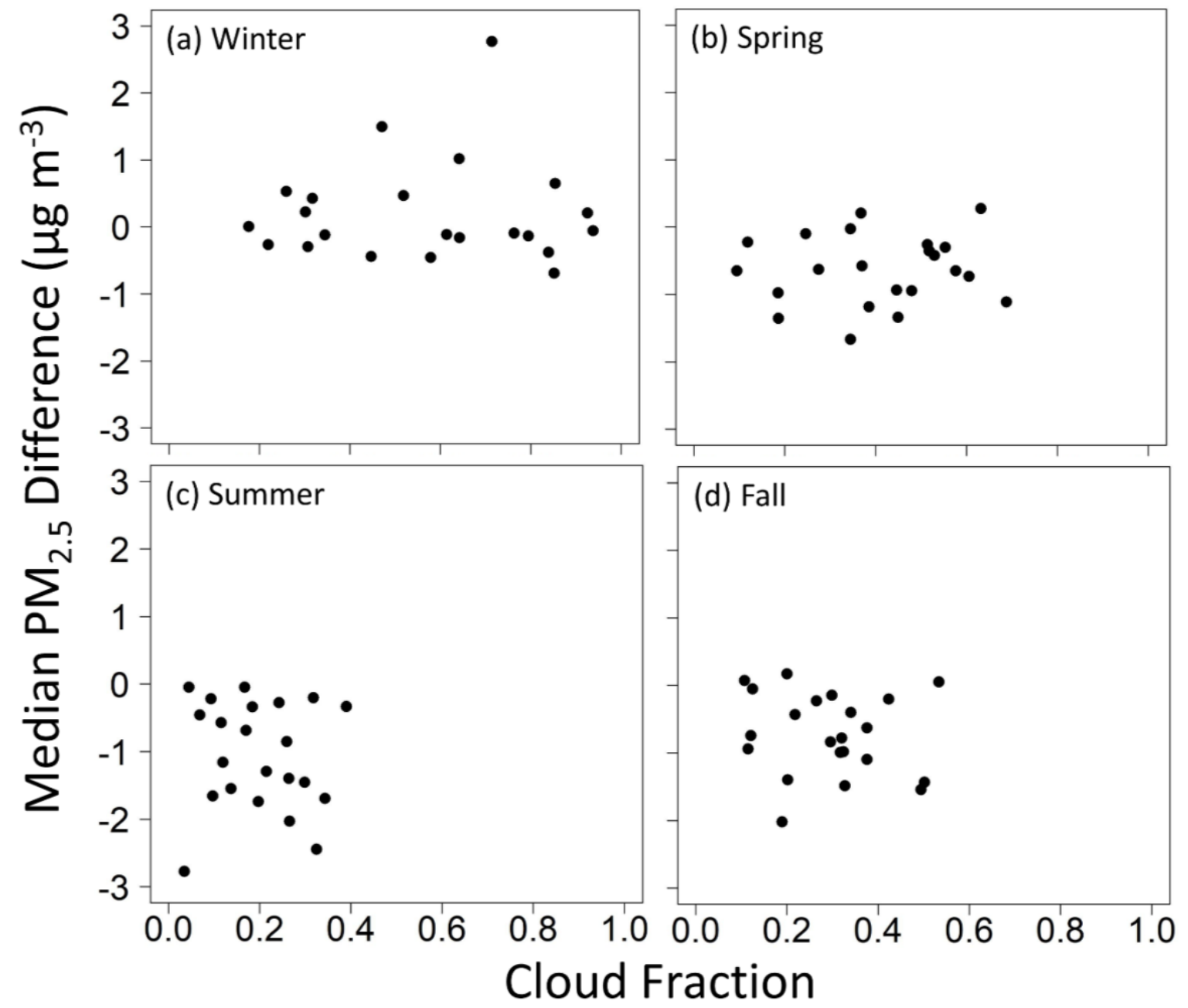

Fig. S7. Median Cloudy - Clear Sky differences by region (dots) in PM2.5 by MODIS median cloud fraction for a) winter, b) spring, c) summer, and d) fall. 


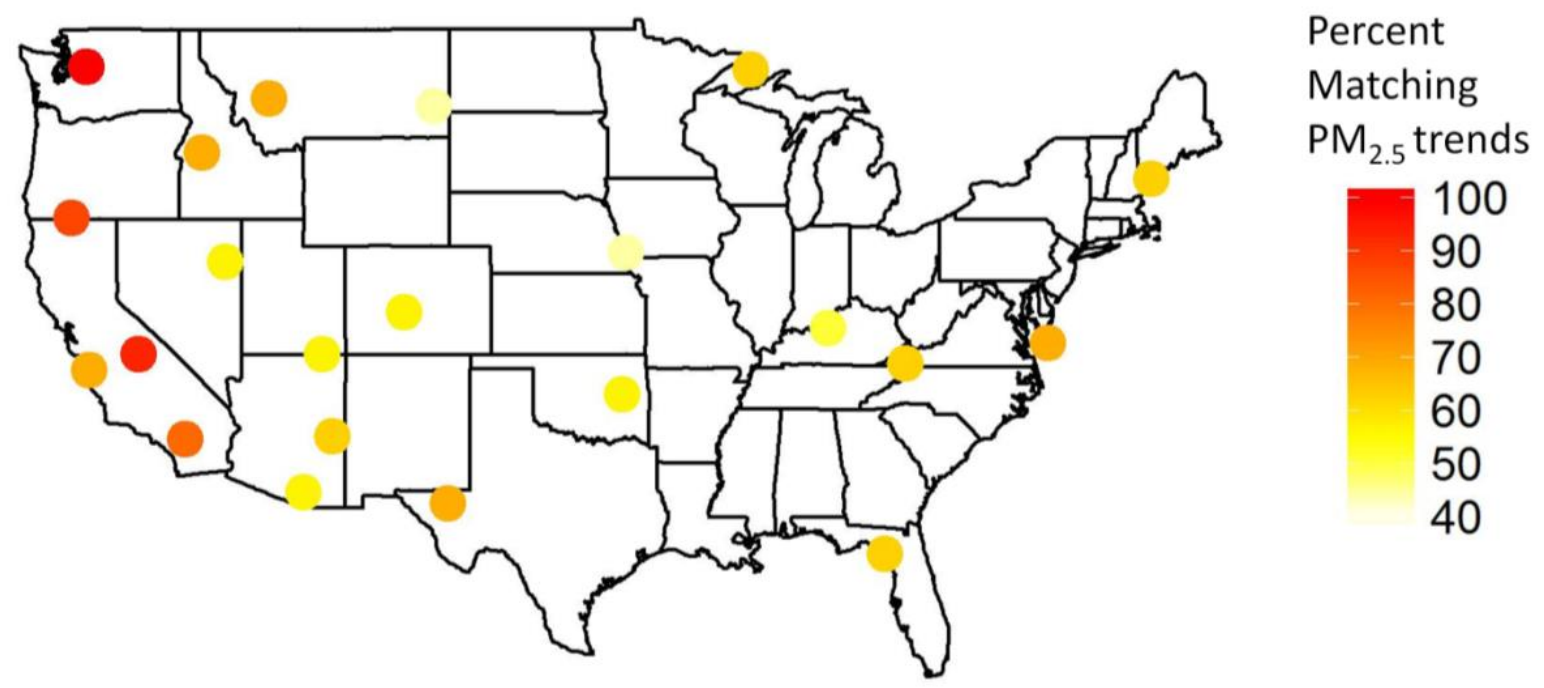

Fig. S8. Percent of $\mathrm{PM}_{2.5}$ chemical constituents ( $\left.\mathrm{SO}_{42-}, \mathrm{NO}_{3-}, \mathrm{TOC}, \mathrm{ALW}\right)$ in which cloudy-clear sky directional trends are the same as those of $\mathrm{PM}_{2.5}$ in each region across the CONUS. For example, if median daily TOC is greater during Cloudy times, and median daily PM2.5 is also greater during Cloudy times, this is counted as a match. 

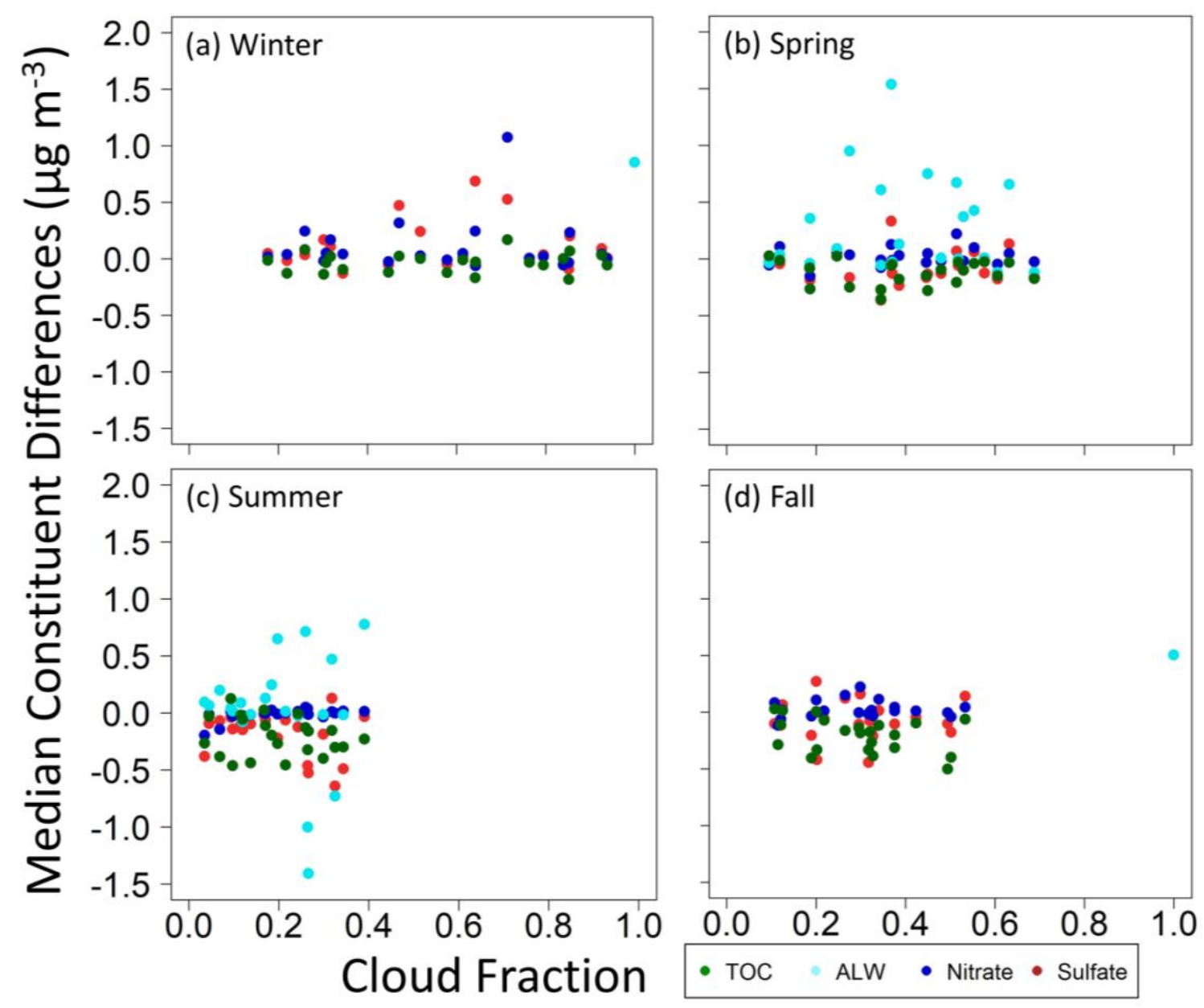

Fig. S9. Median Cloudy - Clear Sky differences by region (dots) in particle chemical constituents (colors) by MODIS median cloud fraction for a) winter, b) spring, c) summer, and d) fall. In each plot, $\mathrm{SO}_{42}$ - is represented by red, $\mathrm{NO}_{3}$ - by dark blue, ALW by light blue, and TOC by green. 


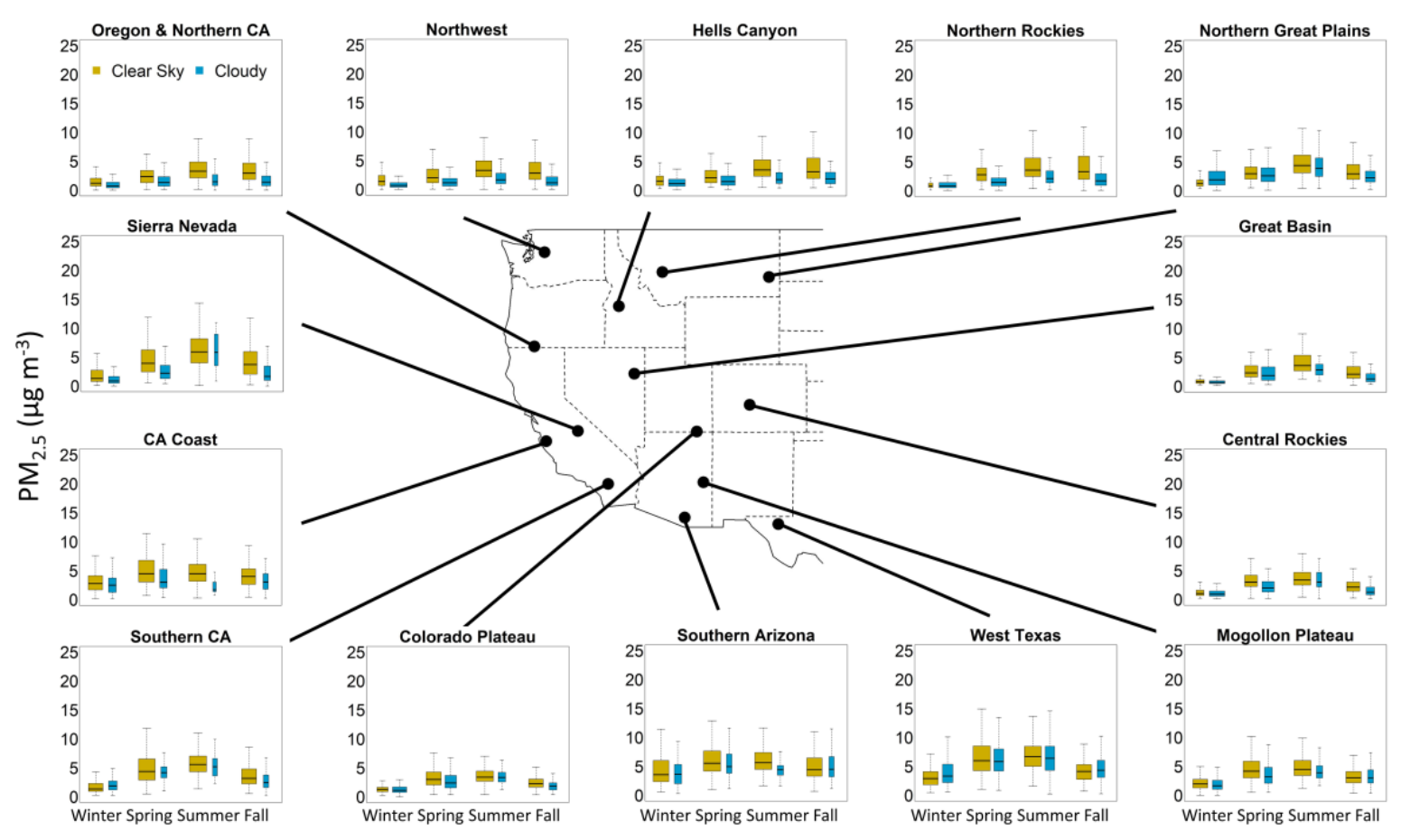

Fig. S10. PM2.5 mass concentrations in the Western U.S. Clear sky values are yellow and Cloudy are blue. Median values are shown as midline with box boundaries as the 75 th and 25 th percentiles. Whiskers are 90th and 10th percentiles. Outliers are not shown but are included in calculations. PM2.5 mass is typically highest during Clear Sky conditions with the exception of winter. 


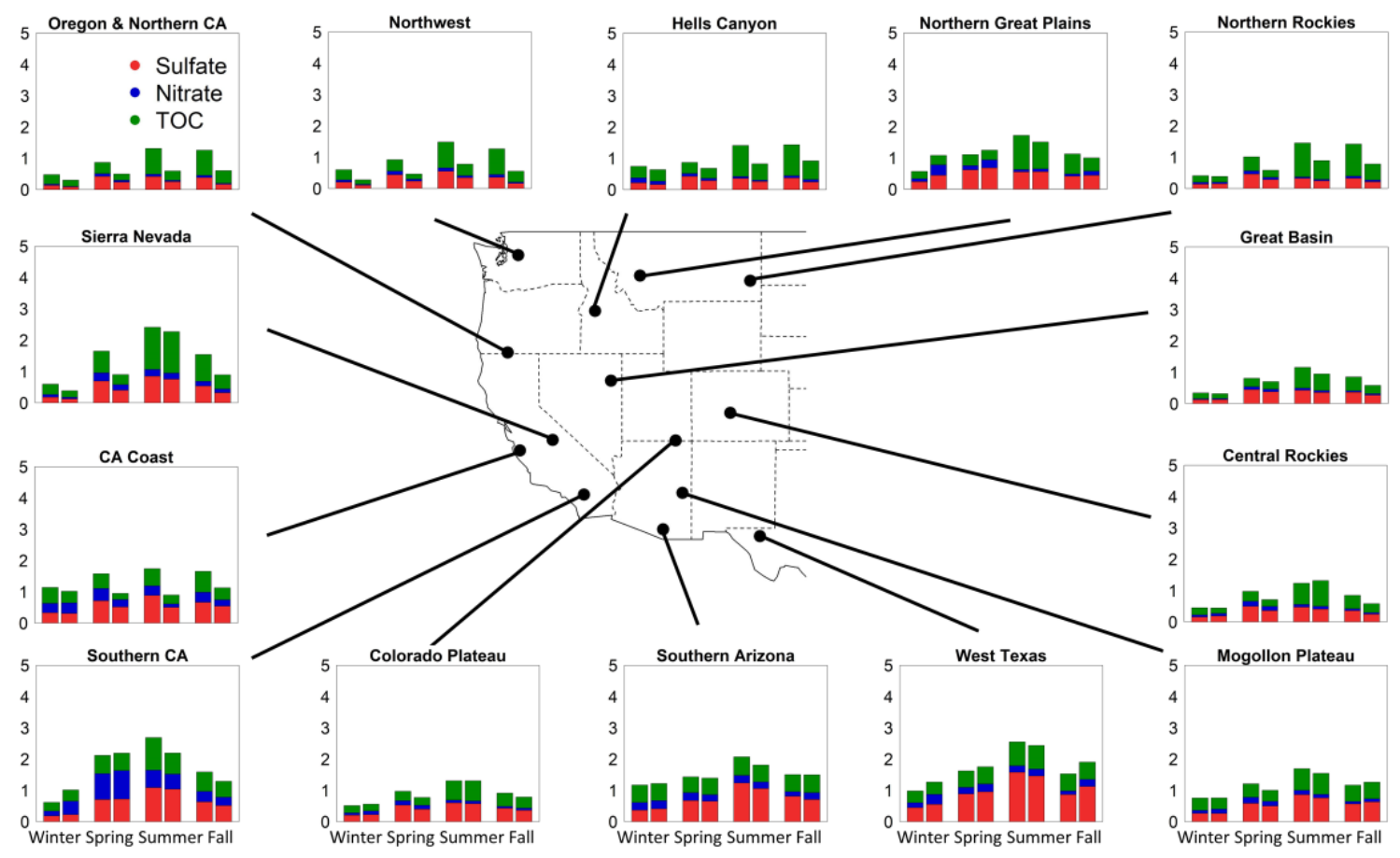

Figure S11. Seasonal median values of $\mathrm{PM}_{2.5}$ chemical constituent in the Western U.S. during MODIS-defined Clear sky (left stacked bar in each pair) and Cloudy (right stacked bar in each pair) conditions. Total organic carbon mass concentration are nearly universally higher during Clear Sky conditions in all regions and this pattern is unique among PM2.5 chemical constituents. 


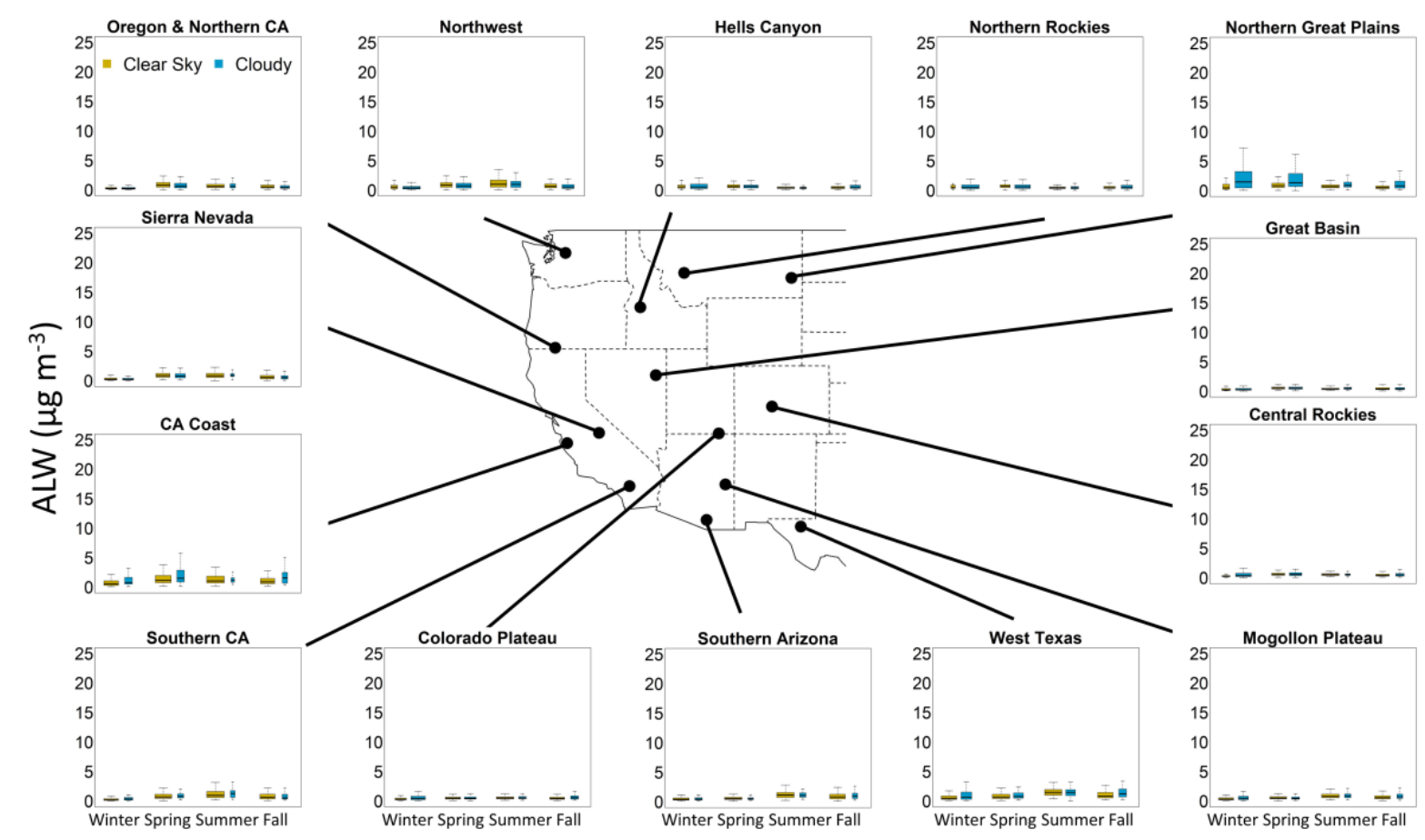

Figure S12. Distributions in ALW mass concentrations during Clear Sky (yellow) and Cloudy (blue) times in the Western U.S. Note y-axis scale is drawn to be consistent with values for the Eastern U.S. presented in main text. Median values are shown as midline with box boundaries as the 75 th and 25 th percentiles. Whiskers are 90 th and 10 th percentiles. Outliers are not shown but are included in calculations. 
Table S1. Variable input conditions to ISORROPIA-II for each water uptake scenario.

\begin{tabular}{|c|c|c|c|}
\hline & "Clear Sky" & "Cloudy" & "Mixed" \\
\hline $\begin{array}{c}\text { Meteorology } \\
\text { MODIS classification - Clear Sky }\end{array}$ & $\sqrt{ }$ & & \\
\hline $\begin{array}{c}\text { Meteorology } \\
\text { MODIS classification - Cloudy }\end{array}$ & & $\sqrt{ }$ & $\sqrt{ }$ \\
\hline $\begin{array}{c}\text { PM2.5 Chemical Composition } \\
\text { MODIS classification - Clear Sky }\end{array}$ & $\sqrt{ }$ & & $\sqrt{ }$ \\
\hline $\begin{array}{l}\text { PM2.5 Chemical Composition } \\
\text { MODIS classification - Cloudy }\end{array}$ & & $\sqrt{ }$ & \\
\hline
\end{tabular}


Table S2. Seasonal differences in reported PM2.5 mass concentration medians (cloudy-clear sky) in all chemical climatology regions. Negative values indicate that clear sky concentrations are larger than cloudy. Bold and italicized values are significantly different by the Mann-Whitney U Test.

\begin{tabular}{|c|c|c|c|c|}
\hline Region & Spring & Summer & Fall & Winter \\
\hline Northwest & -0.73 & -1.46 & -1.44 & -0.69 \\
\hline OR/NorCal & -0.94 & -1.66 & -1.49 & -0.46 \\
\hline CA Coast & -1.36 & -2.77 & -0.94 & -0.27 \\
\hline Sierra NV & -1.67 & -0.05 & -2.02 & -0.44 \\
\hline SoCal & -0.23 & -0.46 & -0.75 & 0.53 \\
\hline HellsCyn & -0.65 & -1.55 & -1.10 & -0.38 \\
\hline Great Basin & -0.36 & -0.69 & -0.78 & -0.10 \\
\hline N Rockies & -1.12 & -1.30 & -1.55 & -0.06 \\
\hline CO Plateau & -0.58 & -0.05 & -0.43 & -0.12 \\
\hline Mogollon Plateau & -0.98 & -0.57 & -0.06 & -0.30 \\
\hline Southern AZ & -0.65 & -1.16 & 0.07 & 0.00 \\
\hline N Great Plains & -0.30 & -0.34 & -0.63 & 0.65 \\
\hline Central Rockies & -0.95 & -0.22 & -0.84 & -0.14 \\
\hline West TX & -0.10 & -0.28 & 0.17 & 0.42 \\
\hline Central Great Plains & -0.26 & -0.85 & -0.15 & 2.76 \\
\hline Mid South & 0.20 & -1.74 & -0.23 & 1.49 \\
\hline Boundary Waters & 0.27 & -0.20 & 0.05 & 0.20 \\
\hline OH River Valley & -1.34 & -1.70 & -0.40 & 1.02 \\
\hline Appalachia & -1.19 & -2.45 & -0.99 & 0.46 \\
\hline Southeast & -0.63 & -2.03 & -1.40 & 0.22 \\
\hline E Coast & -0.03 & -1.40 & -0.99 & -0.12 \\
\hline Northeast & -0.42 & -0.33 & -0.21 & -0.16 \\
\hline
\end{tabular}


Table S3. Seasonal all sky (clear and cloudy data) and clear sky differences in PM2.5 mass concentration medians (all sky-clear sky) in all chemical climatology regions. Negative values indicate that clear sky PM2.5 concentrations are larger than all sky. Bold and italicized values are significantly different by the Mann-Whitney U Test.

\begin{tabular}{ccccc}
\hline Region & Spring & Summer & Fall & Winter \\
\hline Northwest & $\mathbf{- 0 . 4 7}$ & $\mathbf{- 0 . 4 0}$ & $\mathbf{- 0 . 6 8}$ & $\mathbf{- 0 . 6 5}$ \\
OR/NorCal & $\mathbf{- 0 . 4 6}$ & $\mathbf{- 0 . 1 4}$ & $\mathbf{- 0 . 5 2}$ & $\mathbf{- 0 . 2 7}$ \\
CA Coast & -0.21 & -0.07 & -0.14 & -0.05 \\
Sierra NV & $\mathbf{- 0 . 6 7}$ & -0.01 & $\mathbf{- 0 . 3 6}$ & $\mathbf{- 0 . 2 1}$ \\
SoCal & -0.08 & -0.04 & -0.03 & 0.11 \\
HellsCyn & $\mathbf{- 0 . 4 2}$ & -0.18 & $\mathbf{- 0 . 4 5}$ & -0.31 \\
Great Basin & -0.12 & -0.13 & $\mathbf{- 0 . 2 2}$ & -0.07 \\
N Rockies & $\mathbf{- 0 . 8 2}$ & $\mathbf{- 0 . 2 9}$ & $\mathbf{- 0 . 9 1}$ & -0.05 \\
CO Plateau & $\mathbf{- 0 . 2 2}$ & -0.01 & $\mathbf{- 0 . 0 8}$ & -0.06 \\
Mogollon Plateau & $\mathbf{- 0 . 1 6}$ & -0.06 & -0.01 & -0.09 \\
Southern AZ & -0.01 & -0.15 & 0.02 & 0.00 \\
N Great Plains & $\mathbf{- 0 . 1 5}$ & -0.06 & $\mathbf{- 0 . 2 7}$ & $\mathbf{0 . 4 2}$ \\
Central Rockies & $\mathbf{- 0 . 3 6}$ & -0.03 & $\mathbf{- 0 . 2 4}$ & -0.11 \\
West TX & -0.02 & -0.13 & 0.02 & 0.13 \\
Central Great Plains & -0.14 & -0.23 & -0.05 & $\mathbf{1 . 6 9}$ \\
Mid South & 0.12 & -0.40 & -0.05 & $\mathbf{0 . 5 8}$ \\
Boundary Waters & 0.11 & -0.05 & 0.02 & 0.19 \\
OH River Valley & -0.78 & $\mathbf{- 0 . 7 2}$ & -0.17 & 0.67 \\
Appalachia & $\mathbf{0 . 4 2}$ & $\mathbf{- 0 . 7 8}$ & $\mathbf{- 0 . 3 0}$ & 0.22 \\
Southeast & -0.19 & $\mathbf{- 0 . 7 4}$ & $\mathbf{- 0 . 3 4}$ & 0.07 \\
E Coast & -0.01 & -0.36 & -0.18 & -0.05 \\
Northeast & $\mathbf{- 0 . 2 1}$ & $\mathbf{- 0 . 1 2}$ & -0.11 & -0.08 \\
\hline
\end{tabular}


Table S4. Seasonal differences in $\mathrm{SO}_{42-}$ mass concentration medians (cloudy-clear sky) in all chemical climatology regions. Negative values indicate that clear sky $\mathrm{SO}_{42-}$ concentrations are larger than cloudy. Bold and italicized values are significantly different by the Mann-Whitney U Test.

\begin{tabular}{|c|c|c|c|c|}
\hline Region & Spring & Summer & Fall & Winter \\
\hline Northwest & -0.18 & -0.19 & -0.18 & -0.09 \\
\hline OR/NorCal & -0.17 & -0.14 & -0.21 & -0.05 \\
\hline CA Coast & -0.19 & -0.38 & -0.12 & -0.02 \\
\hline Sierra NV & -0.27 & -0.09 & -0.21 & -0.05 \\
\hline SoCal & -0.04 & -0.07 & -0.12 & 0.03 \\
\hline HellsCyn & -0.13 & -0.10 & -0.11 & -0.05 \\
\hline Great Basin & -0.06 & -0.06 & -0.08 & -0.01 \\
\hline N Rockies & -0.16 & -0.06 & -0.10 & 0.01 \\
\hline CO Plateau & -0.13 & -0.01 & -0.06 & 0.02 \\
\hline Mogollon Plateau & -0.09 & -0.11 & 0.06 & -0.01 \\
\hline Southern AZ & -0.03 & -0.15 & -0.10 & 0.04 \\
\hline N Great Plains & 0.06 & 0.02 & 0.04 & 0.20 \\
\hline Central Rockies & -0.13 & -0.04 & -0.11 & 0.03 \\
\hline West TX & 0.08 & -0.12 & 0.27 & 0.11 \\
\hline Central Great Plains & 0.07 & 0.00 & 0.16 & 0.52 \\
\hline Mid South & 0.33 & -0.22 & 0.12 & 0.47 \\
\hline Boundary Waters & 0.13 & 0.13 & 0.14 & 0.09 \\
\hline OH River Valley & -0.14 & -0.49 & 0.01 & 0.68 \\
\hline Appalachia & -0.24 & -0.64 & -0.17 & 0.24 \\
\hline Southeast & -0.17 & -0.53 & -0.42 & 0.17 \\
\hline E Coast & -0.37 & -0.46 & -0.44 & -0.13 \\
\hline Northeast & -0.10 & -0.03 & -0.05 & -0.05 \\
\hline
\end{tabular}


Table S5. Seasonal differences in $\mathrm{NO}_{3}$ - mass concentration medians (cloudy-clear sky) in all chemical climatology regions. Negative values indicate that clear sky $\mathrm{NO}_{3}$ - concentrations are larger than cloudy. Bold and italicized values are significantly different by the Mann-Whitney U Test.

\begin{tabular}{|c|c|c|c|c|}
\hline Region & Spring & Summer & Fall & Winter \\
\hline Northwest & -0.05 & -0.03 & -0.04 & -0.04 \\
\hline OR/NorCal & -0.03 & -0.03 & -0.03 & -0.01 \\
\hline CA Coast & -0.15 & -0.20 & -0.12 & 0.04 \\
\hline Sierra NV & -0.08 & -0.02 & -0.04 & -0.03 \\
\hline SoCal & 0.11 & -0.14 & -0.06 & 0.24 \\
\hline HellsCyn & -0.02 & -0.02 & 0.01 & -0.06 \\
\hline Great Basin & 0.00 & -0.01 & 0.00 & 0.00 \\
\hline N Rockies & -0.03 & 0.00 & -0.01 & 0.00 \\
\hline CO Plateau & -0.02 & 0.00 & 0.01 & 0.04 \\
\hline Mogollon Plateau & -0.04 & -0.02 & 0.02 & 0.05 \\
\hline Southern AZ & -0.06 & -0.02 & 0.08 & 0.01 \\
\hline N Great Plains & 0.10 & 0.02 & 0.04 & 0.23 \\
\hline Central Rockies & -0.02 & 0.01 & -0.01 & 0.02 \\
\hline West TX & 0.05 & 0.01 & 0.11 & 0.17 \\
\hline Central Great Plains & 0.22 & 0.05 & 0.22 & 1.07 \\
\hline Mid South & 0.13 & -0.01 & 0.15 & 0.32 \\
\hline Boundary Waters & 0.05 & 0.01 & 0.04 & 0.04 \\
\hline OH River Valley & 0.05 & 0.01 & 0.11 & 0.24 \\
\hline Appalachia & 0.03 & 0.00 & 0.01 & 0.02 \\
\hline Southeast & 0.03 & -0.02 & 0.00 & -0.02 \\
\hline E Coast & -0.01 & 0.04 & -0.02 & 0.04 \\
\hline Northeast & -0.02 & 0.01 & 0.01 & -0.06 \\
\hline
\end{tabular}


Table S6. Seasonal differences in TOC mass concentration medians (cloudy-clear sky) in all chemical climatology regions. Negative values indicate that clear sky TOC concentrations are larger than cloudy. Bold and italicized values are significantly different by the Mann-Whitney U Test.

\begin{tabular}{|c|c|c|c|c|}
\hline Region & Spring & Summer & Fall & Winter \\
\hline Northwest & -0.15 & -0.40 & -0.40 & -0.18 \\
\hline OR/NorCal & -0.15 & -0.46 & -0.39 & -0.12 \\
\hline CA Coast & -0.27 & -0.27 & -0.29 & -0.13 \\
\hline Sierra NV & -0.36 & -0.03 & -0.41 & -0.12 \\
\hline SoCal & -0.01 & -0.38 & -0.11 & 0.08 \\
\hline HellsCyn & -0.03 & -0.44 & -0.32 & 0.00 \\
\hline Great Basin & -0.03 & -0.11 & -0.17 & -0.03 \\
\hline N Rockies & -0.18 & -0.46 & -0.50 & -0.06 \\
\hline CO Plateau & -0.05 & 0.03 & -0.07 & -0.01 \\
\hline Mogollon Plateau & -0.08 & -0.02 & 0.01 & -0.04 \\
\hline Southern AZ & 0.02 & -0.06 & 0.03 & -0.01 \\
\hline N Great Plains & -0.04 & -0.20 & -0.20 & 0.07 \\
\hline Central Rockies & -0.10 & 0.13 & -0.14 & -0.06 \\
\hline West TX & 0.02 & -0.01 & 0.00 & 0.02 \\
\hline Central Great Plains & -0.21 & -0.13 & -0.18 & 0.17 \\
\hline Mid South & -0.06 & -0.27 & -0.16 & 0.02 \\
\hline Boundary Waters & -0.03 & -0.16 & -0.06 & 0.03 \\
\hline OH River Valley & -0.28 & -0.30 & -0.12 & -0.17 \\
\hline Appalachia & -0.18 & -0.30 & -0.26 & 0.00 \\
\hline Southeast & -0.25 & -0.16 & -0.33 & -0.14 \\
\hline E Coast & -0.27 & -0.32 & -0.33 & -0.10 \\
\hline Northeast & -0.10 & -0.23 & -0.10 & -0.03 \\
\hline
\end{tabular}


Table S7. Seasonal differences in ALW and RH medians (cloudy-clear sky) in all chemical climatology regions. Negative values indicate that clear sky temperatures are larger than cloudy. Bold and italicized values are significantly different by the Mann-Whitney U Test.

\begin{tabular}{|c|c|c|c|c|c|c|c|c|}
\hline Region & \multicolumn{2}{|c|}{ Spring } & \multicolumn{2}{|c|}{ Summer } & \multicolumn{2}{|c|}{ Fall } & \multicolumn{2}{|c|}{ Winter } \\
\hline & ALW & $\mathrm{RH}$ & ALW & $\mathrm{RH}$ & ALW & $\mathrm{RH}$ & ALW & $\mathrm{RH}$ \\
\hline Northwest & -0.12 & 0.12 & -0.02 & 0.13 & -0.09 & 0.22 & -0.12 & 0.08 \\
\hline OR/NorCal & -0.15 & 0.13 & 0.01 & 0.26 & -0.05 & 0.30 & 0.00 & 0.19 \\
\hline CA Coast & 0.35 & 0.21 & 0.09 & 0.39 & 0.66 & 0.28 & 0.24 & 0.21 \\
\hline Sierra NV & -0.06 & 0.23 & 0.06 & 0.09 & -0.03 & 0.24 & -0.01 & 0.15 \\
\hline SoCal & 0.04 & 0.03 & 0.20 & 0.16 & -0.09 & 0.10 & 0.16 & 0.23 \\
\hline HellsCyn & 0.01 & 0.13 & -0.01 & 0.20 & 0.07 & 0.25 & -0.02 & 0.06 \\
\hline Great Basin & 0.00 & 0.12 & 0.13 & 0.11 & 0.02 & 0.15 & 0.01 & 0.08 \\
\hline N Rockies & -0.12 & 0.13 & 0.01 & 0.14 & 0.06 & 0.21 & -0.01 & 0.00 \\
\hline CO Plateau & -0.03 & 0.08 & 0.02 & 0.05 & 0.14 & 0.18 & 0.15 & 0.15 \\
\hline $\begin{array}{l}\text { Mogollon } \\
\text { Plateau }\end{array}$ & -0.04 & 0.05 & 0.09 & 0.08 & 0.15 & 0.11 & 0.15 & 0.15 \\
\hline Southern AZ & -0.04 & 0.01 & -0.08 & 0.11 & 0.15 & 0.07 & 0.05 & 0.00 \\
\hline $\begin{array}{l}\text { N Great } \\
\text { Plains }\end{array}$ & 0.42 & 0.18 & 0.25 & 0.11 & 0.21 & 0.15 & 1.01 & 0.12 \\
\hline $\begin{array}{l}\text { Central } \\
\text { Rockies }\end{array}$ & 0.01 & 0.15 & 0.04 & 0.08 & 0.04 & 0.17 & 0.14 & 0.14 \\
\hline West TX & 0.09 & 0.03 & -0.03 & 0.01 & 0.38 & 0.09 & 0.22 & 0.02 \\
\hline $\begin{array}{l}\text { Central Great } \\
\text { Plains }\end{array}$ & 0.67 & 0.10 & 0.71 & 0.11 & 0.59 & 0.12 & 2.48 & 0.14 \\
\hline Mid South & 1.54 & 0.14 & 0.65 & 0.13 & 1.18 & 0.16 & 2.29 & 0.16 \\
\hline $\begin{array}{l}\text { Boundary } \\
\text { Waters }\end{array}$ & 0.65 & 0.12 & 0.47 & 0.06 & 0.66 & 0.07 & 0.13 & 0.02 \\
\hline $\begin{array}{c}\text { OH River } \\
\text { Valley }\end{array}$ & 0.75 & 0.07 & -0.02 & 0.05 & 1.50 & 0.11 & 4.58 & 0.10 \\
\hline Appalachia & 0.13 & 0.09 & -0.73 & 0.05 & 0.81 & 0.12 & 1.51 & 0.12 \\
\hline Southeast & 0.95 & 0.06 & -1.41 & 0.01 & -0.70 & 0.04 & 1.63 & 0.06 \\
\hline E Coast & 0.61 & 0.10 & -1.00 & 0.04 & 0.40 & 0.08 & 1.35 & 0.11 \\
\hline Northeast & 0.37 & 0.10 & 0.78 & 0.06 & 0.43 & 0.09 & 0.75 & 0.10 \\
\hline
\end{tabular}


Table S8. Seasonal differences in temperature medians (cloudy-clear sky) in all chemical climatology regions. Negative values indicate that clear sky temperatures are larger than cloudy. Bold and italicized values are significantly different by the Mann-Whitney U Test.

\begin{tabular}{|c|c|c|c|c|}
\hline Region & Spring & Summer & Fall & Winter \\
\hline Northwest & -1.65 & -3.69 & -4.60 & -0.41 \\
\hline OR/NorCal & -1.89 & -3.67 & -6.26 & -1.02 \\
\hline CA Coast & -1.42 & -4.41 & -3.40 & 0.54 \\
\hline Sierra NV & -5.91 & 0.44 & -7.64 & -1.65 \\
\hline SoCal & -2.40 & 2.60 & -2.85 & -0.37 \\
\hline HellsCyn & -3.10 & -4.87 & -7.93 & -1.47 \\
\hline Great Basin & -2.50 & -0.13 & -4.99 & -0.65 \\
\hline N Rockies & -4.07 & -4.60 & -7.10 & -2.40 \\
\hline CO Plateau & -4.31 & 1.26 & -5.62 & -4.36 \\
\hline Mogollon Plateau & -3.78 & 0.56 & -3.34 & -2.03 \\
\hline Southern AZ & -0.46 & 0.43 & -4.54 & -0.21 \\
\hline N Great Plains & -4.92 & -2.78 & -7.49 & -4.58 \\
\hline Central Rockies & -6.33 & -1.01 & -7.29 & -4.11 \\
\hline West TX & -0.84 & -0.33 & -0.01 & 0.22 \\
\hline Central Great Plains & -3.42 & -1.82 & -1.94 & -4.10 \\
\hline Mid South & -0.37 & -2.13 & -0.44 & -0.21 \\
\hline Boundary Waters & -5.10 & -0.86 & -4.34 & -5.71 \\
\hline OH River Valley & -0.94 & -1.29 & 0.45 & -1.33 \\
\hline Appalachia & -2.11 & -0.93 & 0.88 & -0.79 \\
\hline Southeast & -1.43 & -0.80 & 0.84 & 0.69 \\
\hline E Coast & -0.72 & -0.85 & 2.06 & 1.97 \\
\hline Northeast & -3.28 & -0.80 & -1.38 & -1.68 \\
\hline
\end{tabular}


Table S9. Cloudy - Clear Sky and Cloudy - Mixed differences in each of the chemical climatology regions that comprise the eastern US. Negative values indicate that Cloudy concentrations are larger.

\begin{tabular}{|c|c|c|c|c|c|}
\hline Season & $\begin{array}{c}\text { Cloudy - } \\
\text { Clear SO42- }\end{array}$ & $\begin{array}{l}\text { Cloudy - } \\
\text { Clear NO3- }\end{array}$ & $\begin{array}{l}\text { Cloudy - } \\
\text { Clear RH } \\
\end{array}$ & $\begin{array}{c}\text { Cloudy - } \\
\text { Clear ALW } \\
\end{array}$ & $\begin{array}{c}\text { Cloudy - } \\
\text { Mixed ALW } \\
\end{array}$ \\
\hline & \multicolumn{5}{|c|}{ Ohio River Valley } \\
\hline Winter & 0.57 & 0.26 & 0.08 & 4.08 & 2.39 \\
\hline Spring & -0.15 & 0.00 & 0.04 & 0.83 & -0.44 \\
\hline Summer & -0.28 & 0.01 & 0.05 & 0.75 & -0.67 \\
\hline \multirow[t]{2}{*}{ Fall } & -0.01 & -0.01 & 0.10 & 1.17 & -0.02 \\
\hline & \multicolumn{5}{|c|}{ Appalachia } \\
\hline Winter & 0.19 & 0.02 & 0.11 & 1.36 & 0.56 \\
\hline Spring & -0.17 & -0.01 & 0.08 & 0.83 & -0.45 \\
\hline Summer & -0.59 & 0.00 & 0.04 & -0.83 & -1.61 \\
\hline \multirow[t]{2}{*}{ Fall } & -0.12 & 0.03 & 0.09 & 0.79 & -0.35 \\
\hline & \multicolumn{5}{|c|}{ Southeast } \\
\hline Winter & 0.16 & -0.03 & 0.06 & 1.74 & 0.56 \\
\hline Spring & -0.21 & -0.02 & 0.06 & 0.89 & -0.61 \\
\hline Summer & -0.41 & -0.03 & 0.01 & -1.12 & -1.20 \\
\hline \multirow[t]{2}{*}{ Fall } & -0.18 & -0.02 & 0.04 & -0.13 & -0.56 \\
\hline & \multicolumn{5}{|c|}{ East Coast } \\
\hline Winter & -0.20 & 0.06 & 0.10 & 2.01 & -0.51 \\
\hline Spring & -0.21 & -0.07 & 0.07 & 0.71 & -0.86 \\
\hline Summer & 0.07 & 0.02 & 0.03 & 1.23 & 0.22 \\
\hline \multirow[t]{2}{*}{ Fall } & -0.14 & 0.02 & 0.08 & 0.35 & -0.50 \\
\hline & \multicolumn{5}{|c|}{ Northeast } \\
\hline Winter & -0.06 & -0.07 & 0.10 & 0.57 & -0.25 \\
\hline Spring & -0.05 & -0.06 & 0.09 & 0.47 & -0.15 \\
\hline Summer & 0.07 & 0.01 & 0.05 & 0.90 & 0.22 \\
\hline Fall & -0.02 & 0.00 & 0.08 & 0.25 & -0.10 \\
\hline
\end{tabular}

